\title{
Sensitive and fast gas sensor for wide variety of applications based on novel differential infrared photoacoustic principle
}

\author{
$\frac{\text { Ismo Kauppinen }}{1}$ (e-mail: ismo.kauppinen@gasera.fi), Arto Branders ${ }^{1}$, Juho Uotila ${ }^{1}$, Jyrki Kauppinen², \\ Tom Kuusela ${ }^{2}$ \\ ${ }^{1}$ Gasera Ltd., Tykistökatu 4, 20520 Turku, Finland \\ ${ }^{2}$ Department of Physics and Astronomy, University of Turku, 20014 Turku, Finland
}

\section{Introduction}

With the increase in the emission of gases from the industries and vehicles, there is a strongly growing need of a solution to monitor the emissions in order to have a cleaner and a greener atmosphere. Furthermore, a fast and sensitive gas sensor with compact size is needed in several applications such as greenhouse gas flux measurements, breath measurements in medical diagnostics, tail pipe measurements in engine development, leak detection and homeland security. There exist a lot of gas measurement solutions, but they do not satisfy the demand of growing effect of the global warming. Moreover the existing technologies are not sensitive, fast, compact and cost efficient enough.

The ultimate aim of the research is to develop and demonstrate a versatile and miniaturized gas sensor sub-system that has the capability to detect the greenhouse gases along with explosive gases, medical markers and chemical agents with orders of magnitude better sensitivity than prior optical measurement methods at the same package volume.

\section{Photoacoustic gas measurement}

The photoacoustic effect is the process of acoustic wave generation in the sample resulting from the absorption of photons. This process was invented already at the end of 1800 century, but first real applications were introduced not until 1960's. Photoacoustic method has since been used to study various chemical and physical phenomena in number of fields. Some of the applications are trace gas analysis, spectroscopy of weak optical transitions, and probing of optically thick sample. To date photoacoustic spectroscopy is widely used in many areas of research and applications.

The basic theory behind photoacoustic detection is rather simple. Light absorbed in a sample will excite a small fraction of the ground state molecular population to higher energy levels. These excited states will subsequently relax through a combination of radiative and non-radiative pathways. The non-radiative part of the excitations will finally generate heat in the localized region of the light beam and produce a pressure wave, which propagates away from the source. This pressure wave can be then detected with a suitable sensor such as a microphone. Photoacoustic detection is unique method since it is a direct monitor of the non-radiative relaxation channel and thus it complements spectroscopic techniques based on absorption and fluorescence. Photoacoustic method is also an extremely sensitive technique, with the ability to detect highly forbidden optical transitions and trace components in a mixture of gases.

The essential components of the apparatus used for photoacoustic gas analysis or spectroscopy are a source of modulated light, a sample cell, a sensor for detecting the acoustic signals, and the signal processing system.

The light source of the photoacoustic detection system is either a broadband IR-source (blackbody radiator) or continuous wave or pulsed laser. When using an IR-source the radiation must be feed to the sample cell through a narrow band-pass filter or monochromator. With conventional lamp it is very simple to generate lot of infrared power (total power can be several watts), but the modulation of the infrared source is clumsy, in practice the only method is to use a mechanical chopper although small-power 
semiconductor infrared emitters, which can be modulated by current, are already available. Unfortunately, the output power of these sources is quite low and the highest modulation frequency is very limited. With lasers the amplitude and especially frequency modulation is easily performed. However, the suitable wavelengths needed for molecular excitations can be difficult to realize when using laser sources. Infrared light emitting diodes (IR-LED) are interesting alternatives. They are not as broadband sources as blackbody radiators since typical spectral width is $1 / 10$ of the centre wavelength, and therefore, in some applications they can be used without any optical filter. The output power of IR-LEDs is still very low, clearly less than $1 \mathrm{~mW}$, and they are available on the wavelength range of $2-7 \mu \mathrm{m}$. The amplitude modulation of IR-LEDs is easy to accomplish and the modulation frequency can be very high.

The sample cell design depends on the type of the light source, but always the main target is to generate acoustic waves and couple the sensor to acoustic excitations as efficiently as possible. All sample cells can be used either in the non-resonant or resonant mode of operation. If the modulation frequency is much lower than the lowest acoustical resonance frequency, the cell is operated in a non-resonant mode. In this case the sound wavelength is much larger than the cell dimensions, thus sound cannot propagate and standing waves cannot form, and the average pressure in the cavity will oscillate along with the modulation frequency.

\section{Condenser and cantilever microphones}

The device coupling to the acoustic field and converting pressure changes to voltage signal is the most critical part of the apparatus when looking for the best sensitivity in trace gas analysis. Microphones normally used in photoacoustic applications can be divided to two main categories: capacitive and optical. Capacitive microphones are either condenser or electret microphones. Optical sensors are based on measuring the movements of either elastic membrane or silicon cantilever using optical beam deflection method or an interferometer [1].

Both condenser and electret microphones have some fundamental limitations, which cannot be overcome. First, to increase the sensitivity of the microphone the gap between the elastic membrane and the back plate cannot be decreased below certain limit. If the gap is very narrow, the gas between the membrane and plate cannot flow freely because of viscous effects and therefore membrane movements are restricted. Secondly, the tension of the membrane cannot either decrease too much: if the membrane is very flexible the dynamic range of the sensor is extremely small. The response of the membrane on the external pressure is also nonlinear due to the fact that the material has to stretch out radially under pressure variations. Finally, the output impedance of condenser and electret microphones is very high, especially at low frequencies, necessitating the use of very high input impedance amplifier for further signal processing. In practice, the noise of best available amplifiers usually exceeds the noise generated from the transducer itself, thus there are no possibilities to further increase the sensitivity of the microphone.

A cantilever does not have the same mechanical restrictions as a membrane when using it for pressure wave detection in photoacoustic applications. A cantilever has two main benefits. First, the string constant of the vibrating cantilever can be quite easily 2 or 3 decades smaller than one of the membrane, and it can be very easily adjusted by changing the dimensions of the cantilever. Secondly, the dynamical range of the cantilever is very large comparing the one of the membrane, i.e., the cantilever can bend even tens of micrometers without any nonlinear effects or restricting effects. The bending can be measured either using an optical beam or interferometer. If a large dynamical range is needed the optical beam method is not applicable and an interferometer should be used. The interferometric measuring has inherently infinite range of dynamics since the bending can be determined over as many wavelengths of the laser as is needed. This large dynamic range is directly related to the concentration range of the trace gas, which can be measured. This is an important feature of any gas detection and measuring system.

\section{Differential measuring concept}

In the long path absorption spectroscopy the light beam penetrates through the sample gas, and the absorption at two or more wavelengths are measured and compared. The sensitivity of this kind of method depends mainly on the absorption length, output power of the light source and the response of the detector. In practice the absorption path length cannot be increased too much since then, because of 
the exponential decreasing of the signal, the signal-to-noise ratio would be very small, and with wet gases the water absorption can be serious problem.

In the differential photoacoustic detection the selectivity of the traditional absorption method and the high sensitivity of the photoacoustic detector are combined. The basic structure of this system is shown in Fig. 1 (the upper panel). The device consists of three cells: the sample cell containing the unknown concentration of the gas to be detected, the reference cell filled with pure nitrogen and the differential photoacoustic cell containing high concentration of the analysed gas. The differential cell is divided two equal parts and the cantilever is between them, and the bending of the cantilever is measured by using the interferometer. If there is a pressure difference over the cantilever it bends proportionally to that difference, i.e., it acts like a highly sensitive differential pressure sensor. The infrared radiation is directed to the sample and reference cell with an equal power. From these cells the radiation enters to the both parts of the differential cell. The pressure difference can be generated only if the light absorption is different on the both sides of the cantilever, and this is possible only if there have been some absorption of light in the sample cell at the same wavelength specific to the analysed gas. Thus the pressure difference and the bending of the cantilever are directly related to the concentration of the gas.

The differential setup is very flexible. First, the selectivity of the method over various gases is achieved by using suitable gas in the differential cell. Even gas mixtures are possible, and then the output signal depends on the total concentration of these gases in the sample cell. There are several alternatives for he infrared light source, as shown in Figure 1. When using blackbody radiator the beam must be first divided in to two equal parts and then amplitude modulated by a mechanical chopper (Fig 1.the lower left panel). Similar optical design is needed when using laser sources (Fig.1, the upper panel). IR-LEDs as a source is a most compact solution since then it is possible to use two LEDs and balance the system by adjusting the currents of the LEDs (Fig.1, the lower right panel). In the case of laser source (and partially also with IR-LEDs) the selectivity is mainly set by the choice of the wavelength of the source, thus the differential setup does not produce any additional benefits on this feature. However, the sample cell is not part of the photoacoustic system, and therefore, it can be in open path operation mode. If a closed sample cell is used the gas can be changed very fast since there is no risk to damage the microphone as in the photoacoustic cell. In practice this means that the response time of the overall system can be very short.
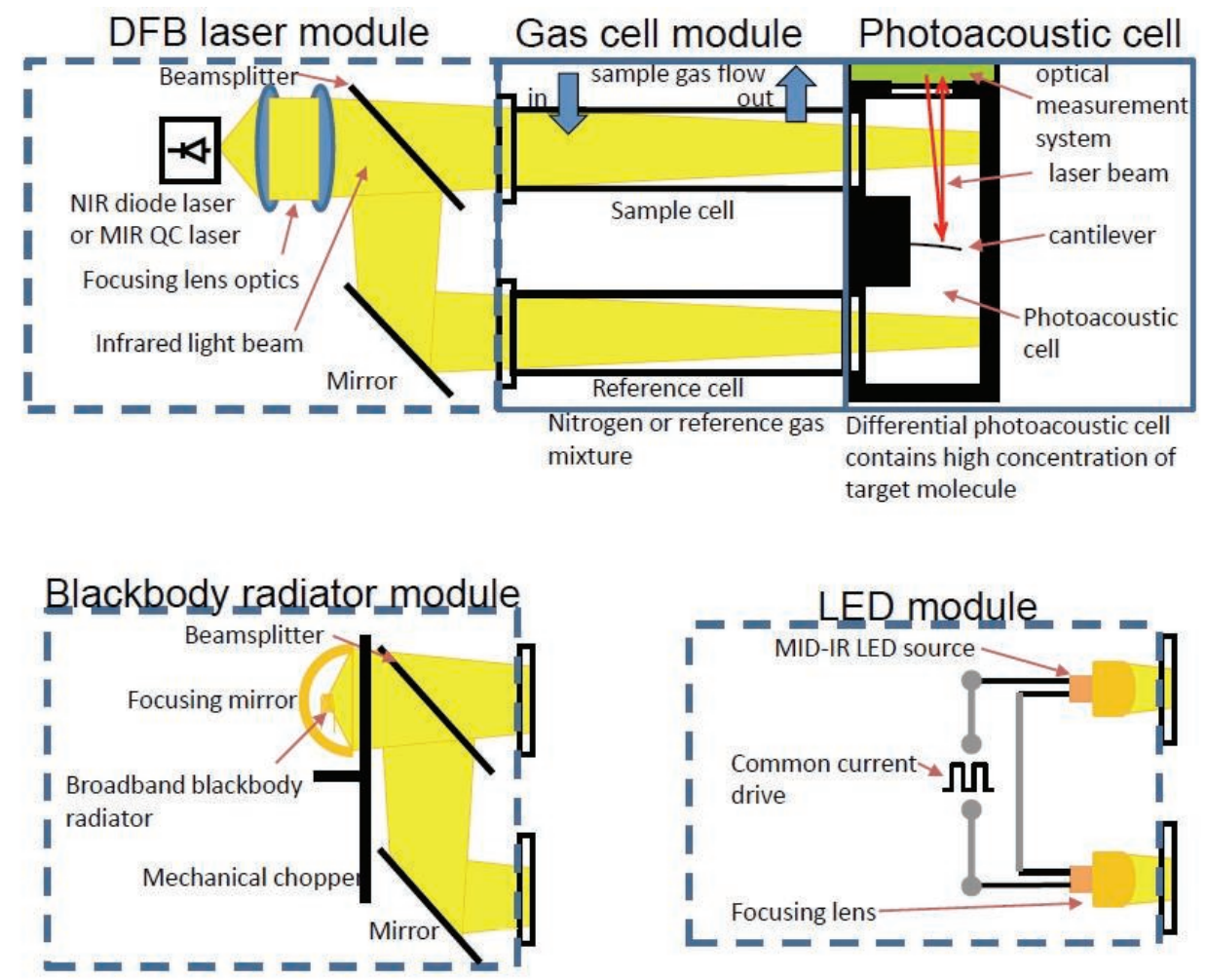

Figure 1. Modular concept of the proposed differential infrared photoacoustic gas sensor. 


\section{Example systems}

One realization of the modular differential system is presented in Fig. 2. Experiments have been done using a DFB laser with a center wavelength $2702,86 \mathrm{~nm}$, power $1 \mathrm{~mW}$ and wavelength modulation at 70 $\mathrm{Hz}$. The laser wavelength corresponds to the one absorption peak of $\mathrm{CO}_{2}$. The differential cell was filled with gas mixture of $50 \% \mathrm{CO}_{2}+50 \% \mathrm{Xe}$, and the pressure was $1 \mathrm{~atm}$. The sample was filled in the following sequences: $\mathrm{N}_{2}$ - laboratory air $\left(\mathrm{CO}_{2}\right.$ concentration about $\left.580 \mathrm{ppm}\right)-\mathrm{CO}_{2}$ in the concentration of $1030 \mathrm{ppm}$ (in $\mathrm{N}_{2}$ ) - $\mathrm{N}_{2}$. The gas was changed by pumping continuously through the sample cell and manually changing the input tube to different gas bottles during the measurement. The $\mathrm{CO}_{2}$ concentration as a function of time is presented in Fig. 3. The sampling rate was $10 \mathrm{~Hz}$. It can be seen that the measured $\mathrm{CO}_{2}$ concentration changed quite fast, and the response time is only 0,1 seconds (see the inserted figure in Fig. 3). The fluctuation of the signal results from the actual concentration variations caused by the pumping system.

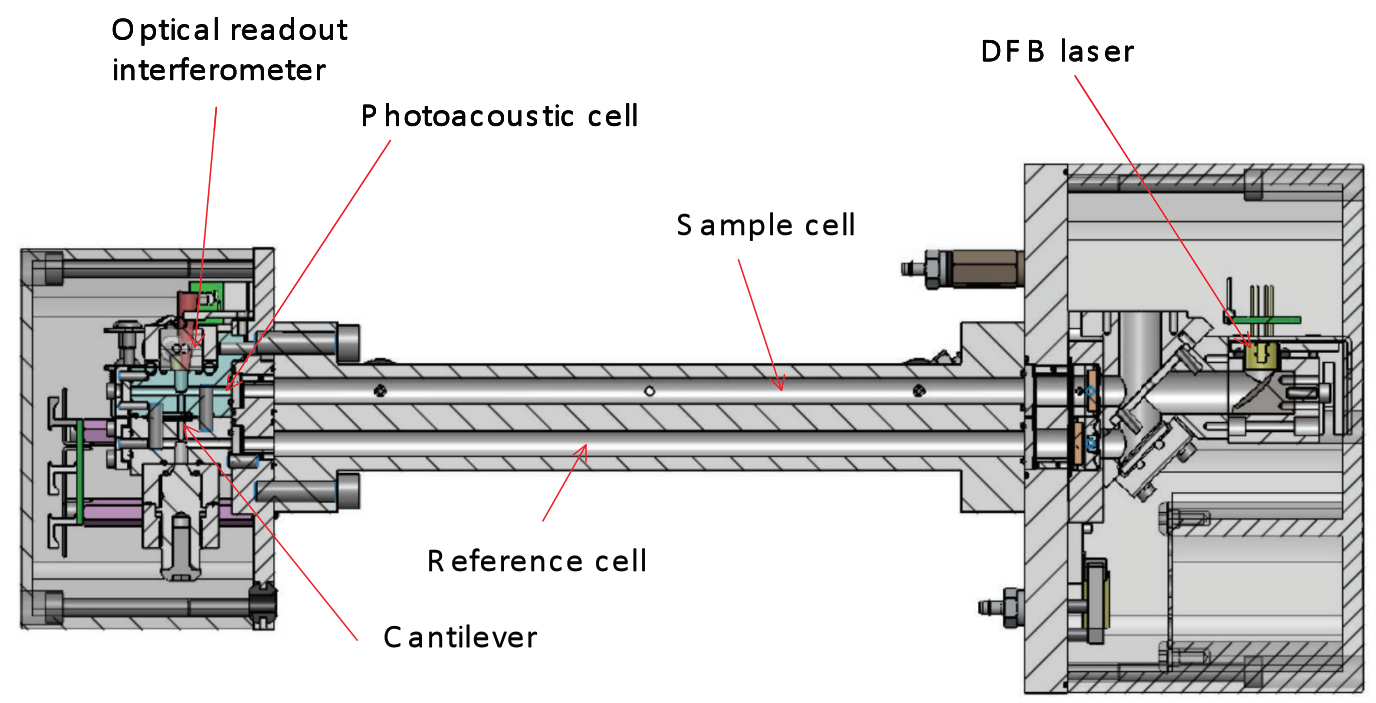

Figure 2. The mechanical structure of the differential system designed for $\mathrm{CO}_{2}$ flux measurements.

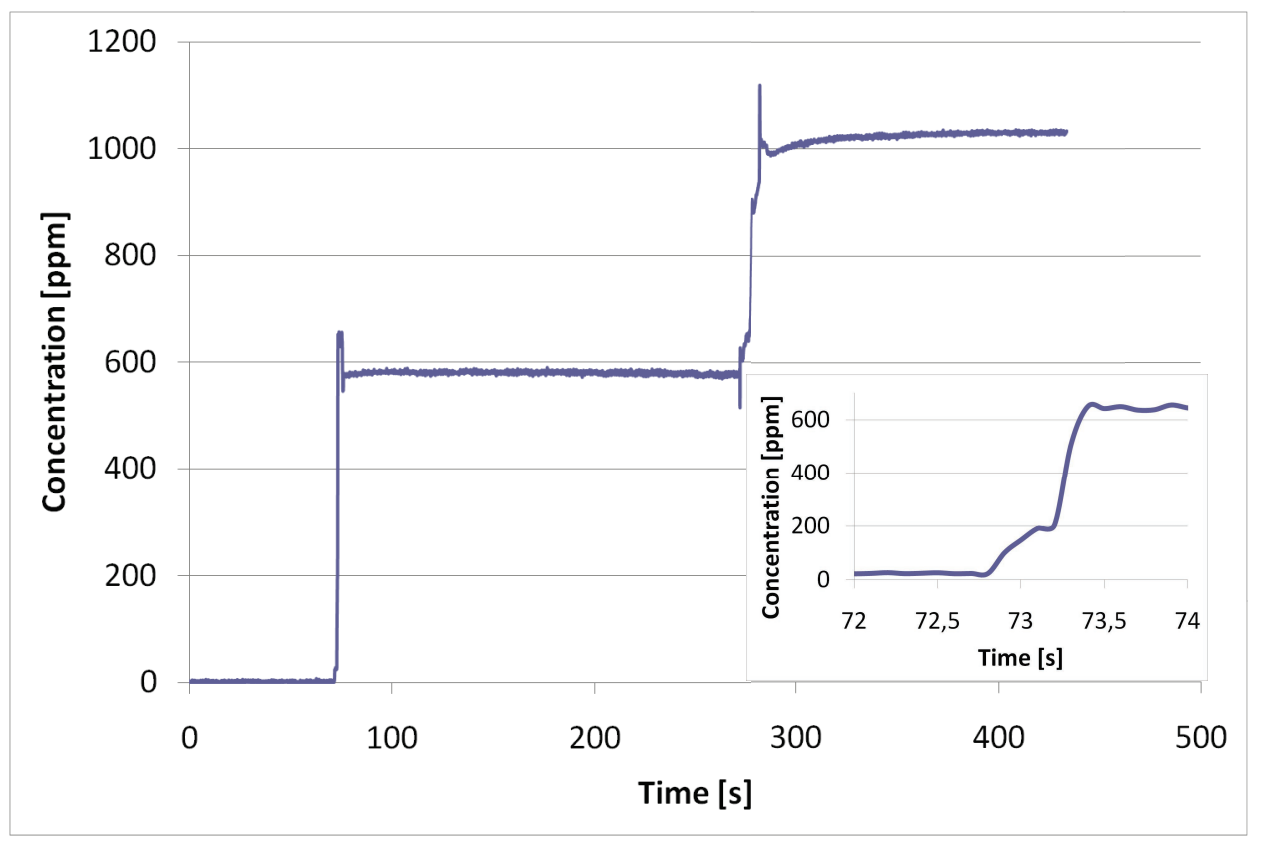

Figure 3. The $\mathrm{CO}_{2}$ concentration as a function of time. 


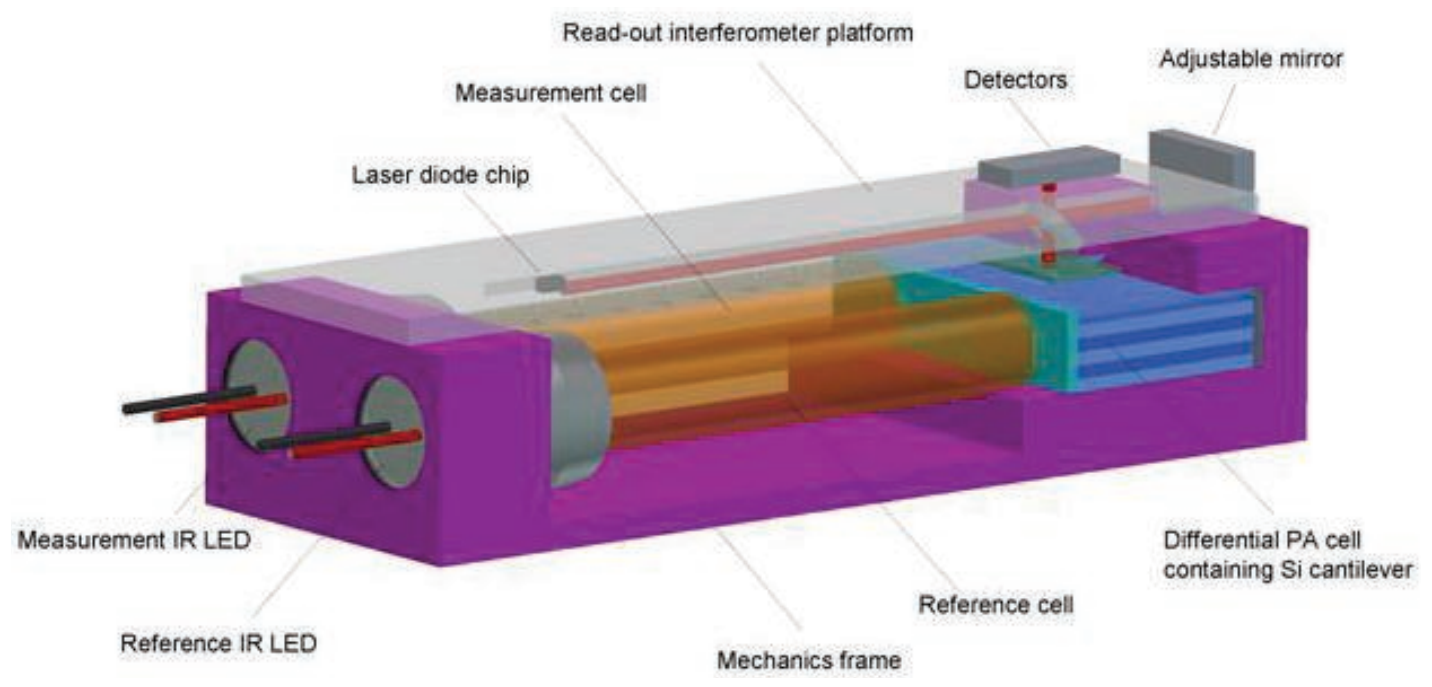

Figure 4. MINIGAS methane leak detector.

In MINIGAS-project (EC FP7 Program \# 224625) another realization of the modular differential photoacoustic system is developed. For a miniaturized solution to methane leak detection an infrared (IR) light-emitting diode (LED) [2] will be designed and utilized resulting in a $5 \mathrm{~cm}^{3}$ packaging volume of the sensor sub-module achieving below ppm detection limit in one second measurement time. The gas cell itself is a flow through cell with few milliliters volume providing the possibility for fast response time. The mechanical design of the device is based on LTCC (Low Temperature Cofired Ceramics) technology, which will be used both for the cell structures and the readout interferometer (see Fig. 4).

This study was supported in part by a grant of the EC FP7 Program (\# 224625, MINIGAS).

[1] T. Kuusela, J. Kauppinen, "Photoacoustic gas analysis using interferometric cantilever microphone", Applied Spectroscopy Reviews, 42, 443-474, (2007).

[2] T. Kuusela, J. Peura, B. A. Matveev, M. A. Remennyy, N. M. Stus', "Photoacoustic gas detection using a cantilever microphone and III-V mid-IR LEDs", Vibrational spectroscopy, 51(2), 289-293 (2009). 